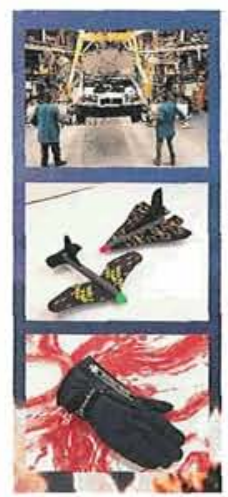

\title{
Monitoring Earth's Atmosphere
}

田 arth's atmosphere is a distinctive blend of chemistry that sustains life here on the planet. With the global population nearing 6 billion, it is believed that human activities are inducing atmospheric change. Because of this linkage, modifications in the atmosphere may be early harbingers of global climate change. To better monitor the atmosphere, from the ground, in the air, and from space, various tools are being employed.

One such tool is a new Micro Pulse Lidar (MPL) System, originally developed by Goddard Space Flight Center and now available commercially from Science \& Engineering Services, Inc. (SESI) of Burtonsville, Maryland through a technology transfer license.

Lidar stands for Light Detection and Ranging, an optical analog to radar. Using a lidar, short, intense pulses of laser light are directed to disperse through the atmosphere. As this laser light propagates, it interacts with aerosols - particles of liquid or solid dispersed as a suspension in gas-and molecular constituents. The backscattered energy is measured by the Lidar system for detailed study.

SESI's Micro Pulse Lidar System is built to characterize numerous details of the composition and dynamics of the atmosphere, such as atmospheric cloud and aerosol concentration. The system is suitable for environmental monitoring studies that require full-time, unattended measurements of cloud and aerosol height structure.

A main feature of SESI's MPL System is beam expansion, making it eye safe at all ranges, including the exit aperture of the instrument. Being eye safe eliminates risk to civilians or aircraft, even in continuous operation. The device has impressive sensitivity in signal detection over a great range. The MPL is capable of detecting all significant cloud and aerosol scattering from the ground through the troposphere, and into the stratosphere-a distance of over 15 miles. Designed for long-term unattended operation, the SESI MPL System is well suited for applications requiring routine monitoring of the atmosphere.

Backscattered radiation received by the MPL is transformed into electrical signals which are subsequently converted into digitized data. Data are then collected, stored, and analyzed by the system's rackmounted IBM-compatible personal computer. The MPL $\mathrm{PC}$ also coordinates all of the system operations.

Another feature of the SESI Lidar is the instrument's modest dimensions, allowing it to be used where space is limited. The system can also be equipped with a protective climate-controlled enclosure, permitting placement of the system in field operations where adequate sheltering facilities do not exist. Upgrading of the unit can be easily done for greater resolution, performance, or to make the instrument more rugged for use in an aircraft.

Studies of climate dynamics, meteorological research, and environmental monitoring are but a few possible applications using SESI's Micro Pulse Lidar.

SESI has been performing Small Business Innovation Research work for Goddard Space Flight Center and Wallops Flight Facility, as well as private industries. SESI is engaged in the development of unique tunable solid state laser systems, various lidar systems for atmospheric measurements, detection and ranging equipment, and medical instrumentation.

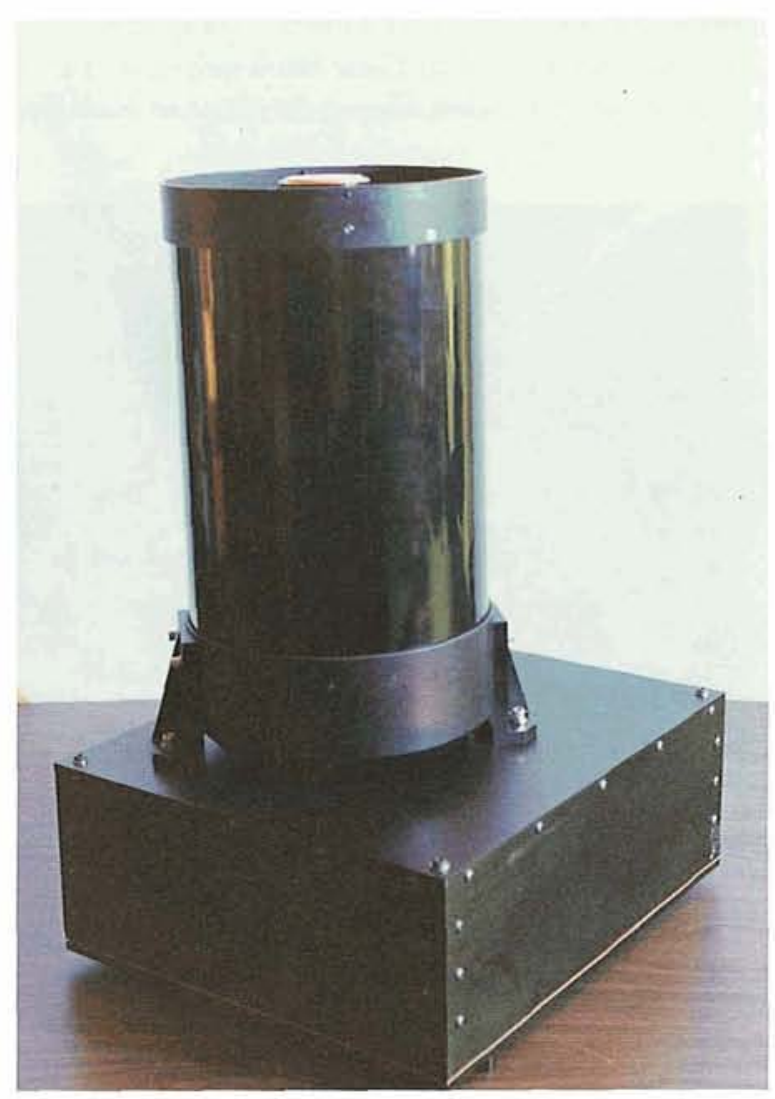

Science and Engineering Services, Inc.'s Micro Pulse Lidar System resulted from NASA research and patents. This instrument is employed to make detailed measurements of atmospheric constituents and is available on the commercial market. 